\title{
Oral Contraceptives after Bariatric Surgery
}

\author{
Joël Schlatter
}

Department of Pharmacy, Hôpital Jean Verdier - APHP, Hôpitaux Universitaires de

Paris-Seine-Saint-Denis, Bondy, France

\section{Key Words}

Bariatric surgery · Oral bioavailability · Drugs · Contraception · Obesity

\section{Abstract}

Objective: Bariatric surgery offers a highly effective mode of treatment for obese patients. Some procedures such as bypass cause an alteration in normal gastrointestinal tract with possible consequences for the uptake of orally administered drugs. Methods: We assessed the literature to ascertain whether the use of oral drugs and especially oral contraceptives is effective and adequate after bariatric surgery. Results: The bioavailability of drugs could be affected by the solubility and $\mathrm{pH}$ of the modified medium after bariatric surgery and by the loss of gastrointestinal transporters. Bariatric surgery could potentially result in a transient change in the absorption of drugs such as analgesics, antibiotics, antiarrhythmics, anticoagulants, psychotropic, and oral contraceptive drugs. Effective contraception is especially critical in the postoperative period, and implants might be representing a safe contraceptive method in women undergoing bariatric surgery. Conclusion: Each drug will have to be evaluated with respect to its site of absorption and its mechanism of absorption, with special attention on parameters influencing the effectiveness of the absorption processes.

(C) 2017 The Author(s)

Published by S. Karger GmbH, Freiburg

\section{Introduction}

The prevalence of obesity doubled worldwide since 1980. In 2014, more than 1.9 billion adults were overweight [1]. Of these, more than 600 million were obese. Overall, approximately $13 \%$ of the world adult populations (11\% of the men and $15 \%$ of the women) were obese in 2014 [1]. In the USA, it was estimated that $36 \%$ of the adult women were obese, with a BMI higher than $40 \mathrm{~kg} / \mathrm{m}^{2}$, and $6 \%$ of the pregnant women suffered from morbid obesity [2-4]. Moreover, the data of the National Health and Nutrition Examination Survey of 2007- 
2008 showed that $10.4 \%$ of the teenagers (12-19 years old) are seriously obese (BMI $\geq 35$ $\mathrm{kg} / \mathrm{m}^{2}$ ] [5]. Epidemiological studies showed that obesity in pregnant women increased significantly the incidence of diabetes, fetal growth restriction, complications of childbirth, prematurity, stillborn babies, and some congenital malformations [6-9]. To reduce these co-morbidities and ensure a significant and sustainable weight loss, bariatric surgery has become one of the preferred procedures over the last years [10-12]. Between 1998 and 2006, the incidence of the bariatric surgery in the USA increased by $800 \%$ (20,000 procedures in $1998 \mathrm{vs.}$ 200,000 procedures in 2006), with $83 \%$ women being in childbearing age [13,14]. Bariatric surgery also improved menstrual disorders and fertility $[14,15]$. The American College of Obstetrical and Gynecology (ACOG) and the French National Authority for Health (HAS) published guidelines for the postoperative management of these patients and recommended taking contraceptives during 12-24 months after the intervention [16, 17]. In France, the national surveys on obesity (ObEpi) published epidemiological data every 3 years [18]. In $2012,32.3 \%$ of adults were in overweight $\left(25<\mathrm{BMI}<30 \mathrm{~kg} / \mathrm{m}^{2}\right)$ and $15 \%$ were obese (BMI $\geq 30 \mathrm{~kg} / \mathrm{m}^{2}$ ). The gold standard surgical weight loss technique is a combination of restrictive and malabsorptive procedures such as gastric bypass (RYGB) and biliopancreatic diversion (BPD) $[19,20]$. The RYGB procedure reduces gastric capacity by $95 \%$ and bypasses the proximal small intestine [21]. Although RYGB was found to be the gold standard procedure, the most commonly performed procedure in many countries is currently the sleeve gastrectomy $[22,23]$. Laparoscopic sleeve gastrectomy is a restrictive procedure that is characterized by the removal of most of the fundus of the stomach without alteration of intestinal absorption; it is considered to be relatively safe with low morbidity [24-26]. The changes of the gastrointestinal tract resulting from such interventions are supposed to also affect the absorption of orally administered drugs. There is a lack of published information regarding the medication used, particularly that of oral contraceptives. Therapeutic failure may occur in significant morbidity and unintended pregnancy. Practitioners involved on the aftercare of patients treated with restrictive and malabsorptive procedures should therefore propose medication alternatives for orally administered drugs. Until pharmacokinetic clinical studies are completed, clinicians should consider the physiochemical properties of drugs, such as the acid dissociation constant ( $\mathrm{pKa}$ ) and the partition coefficient (log P), as well as the localization of intestinal drug transporters.

\section{Factors Affecting the Bioavailability of Drugs}

\section{Solubility and $\mathrm{pH}$}

The rate of dissolution and intrinsic permeability of a drug may be affected by changes in gastrointestinal $\mathrm{pH}$ [27]. The $\mathrm{pH}$ of gastrointestinal fluid has been shown to significantly affect the fraction of the dose absorbed of a drug [28, 29]. Typically, weak base drugs are better dissolved in gastric fluids than in the intestine, whereas weak acid drugs are at a minimum dissolved in the stomach and transported to the less acidic regions of the intestine [30]. The $\mathrm{pH}$ of gastric fluid is typically acidic ranging from 1 to 2 [31]. A reduction of gastric acid secretion in the stomach significantly impaired oral absorption of basic drugs that show low solubility at high $\mathrm{pH}$ due to incomplete dissolution [32,33]. At physiological gastric $\mathrm{pH}$, weak-base drugs are fully charged and have a high solubility in water, whereas weak-acid drugs are fully uncharged and then absorbed more rapidly from the stomach. Weak-base drugs are predominantly absorbed from the intestine because they are uncharged at the intestinal $\mathrm{pH}$ of 8 . The uncharged substances can pass through the mucosal lining of the gastrointestinal tract [19]. The standard bariatric surgery bypass reduces the hydrochloric acid secretion in the stomach and can act on the charge of the drugs depending of their specific 
acid dissociation constant (pKa) [34]. The pKa of a drug is a key physicochemical parameter influencing many biopharmaceutical characteristics. This dissociation constant helps in understanding the charge mode a drug will take across a range of $\mathrm{pH}$. The $\mathrm{pKa}$ influences solubility and permeability, which directly affects absorption. If the pKa of a drug is close to the $\mathrm{pH}$ of the medium, the molecule is charged and its gastrointestinal diffusion is delayed. In summary, increasing gastric $\mathrm{pH}$ should decrease the solubility of basic drugs and increase the solubility of acidic drugs [35].

\section{Gastrointestinal Transporters}

The bypass procedure excludes parts of the intestine from absorption, thus decreasing the number of transporters. The organic anion transporter OATP1A2 is expressed on the duodenum and acts on the diffusion of thyroid hormones, steroid hormones, fluoroquinolones, or statines $[36,37]$. The intestinal oligopeptide PEPT1 transporter interacts with some antibiotics such as beta-lactamines, the angiotensin converting enzyme inhibitors, the thrombin inhibitors, and some antineoplastics [38-41]. The P-glycoprotein (P-gp) plays an important role for the transport of drugs, particularly on the apical surface of the epithelial cells of the jejunum and colon. It also interacts moreover with the diffusion of digoxin, verapamil, diltiazem, and sotalol [42]. P-gp expression level is lowest in the duodenum and highest in the distal ileum and the colon [43]. Cytochromes P450, glucuronyltransferases, sulfotransferases and glutationine-S-transferases are the enzymes present at the enterocytes of the gastrointestinal tract. The cytochrome CYP3A4 is expressed along the entire small intestine, with slightly increased expression from the duodenum to the middle section of the jejunum and a gradually reduced expression in the distal jejunum and ileum [44]. The duodenum and proximal jejunum account for $20-40 \%$ of the total intestinal CYP3A4 activity. Its abundance after RYGB is estimated to decrease by $30 \%$ [45].

\section{Bioavailability of Drugs after Bypass}

\section{Analgesics}

The non-steroidal anti-inflammatory drugs (NSAIDs) have pKa in the range of 3-5 and thus are characterized by poor solubility in the stomach at typical gastric $\mathrm{pH}[46,47]$. These drugs are soluble in the intestine [46]. However, the modification of gastric $\mathrm{pH}$ by RYGB procedures increases the solubility of these drugs with a greater risk of ulcer. The use of paracetamol (pKa of 9.5) or tramadol (pKa 9.4) is regarded as an alternative for the use of NSAIDs in these patients $[48,49]$. As paracetamol is mainly absorbed in the jejunum, this pathway is not affected by RYGB [48]. The plasma concentrations area under the plasma concentration time curves and urinary recovery were similar before and after bypass surgery, and the absorption pattern was essentially the same as in non-obese control subjects [48].

\section{Antibiotics}

Early pharmacokinetic research has shown that some fluoroquinolones are absorbed efficiently from the small intestine and transported by organic anion transporting polypeptides (OATPs) [50]. Colin et al. [51] investigated adequate moxifloxacine concentrations using a population pharmacokinetic analysis and pharmacokinetic-pharmacodynamic simulations in post-bariatric surgery patients. When considering the targets for suppression of bacterial resistance formation, even at minimal inhibition concentration values as low as $0.25 \mathrm{mg} / \mathrm{l}$, standard moxifloxacin dosing does not attain adequate levels in this population. The authors emphasized the need for lean body mass individualized dosing of moxifloxacin. A case of malabsorption after oral administration of amoxicilline and nitrofurantoine exemplified this 
dilemma of gastric bypass in a pregnant patient with urinary tract infection [52]. The urinary tract infection was ultimately resolved by intravenous ceftriaxone injections. A recent study characterized the pharmacokinetics of intravenous and oral linezolid before and 3 months after RYGB surgery [53]. The bioavailability of the drug was not impaired with a mean of 1.14 (0.816-1.47) before and 1.14 (1.01-1.26) after RYGB. The mean AUC with oral linezolid before RYGB was $41.6 \mathrm{mg} . \mathrm{h} / \mathrm{l}$ compared with $98.9 \mathrm{mg} . \mathrm{h} / \mathrm{l}$ after RYGB $(\mathrm{p}<0.001)$. The serum exposure of the drug was more than $50 \%$ lower after bariatric surgery suggesting that dose modification may be needed.

\section{Antiarrhythmics}

Chan et al. [54] compared the pattern and magnitude of oral absorption of digoxin in obese patients before and after RYGB. The median time to peak concentration for digoxin decreased from $40 \mathrm{~min}$ at baseline to 30 and $20 \mathrm{~min}$ at 3 and 12 months after RYGB, respectively. The mean AUC for digoxin, heart rate, and electrocardiogram patterns were similar across the study phases.

\section{Anticoagulants}

A recent study quantified the change in weekly warfarin dose after RYGB in patients requiring long-term warfarin therapy [55]. In the bariatric surgery group, $74.1 \%$ of patients experienced a decrease that was $20 \%$ or more of the preoperative dose compared with $32.2 \%$ of control group patients. This study reported that bariatric surgery could result in a transient increase in warfarin sensitivity manifested clinically by the need for a significant reduction in the postoperative warfarin dose required to achieve therapeutic INR values. The suggested mechanism behind this observation was a more alkaline stomach $\mathrm{pH}$ value resulting in more unionized warfarin available for passive absorption [56].

\section{Psychotropic Drugs}

Many gastric bypass patients have psychiatric illnesses that are treated with medication preoperatively, but there are few data to guide psychiatric drug dosing postoperatively. Chan et al. [54] compared the pattern and magnitude of oral absorption of midazolam in obese patients before and after RYGB. The peak plasma concentration (Cmax) of midazolam increased by $66 \%$ and $71 \%$ at 3 and 12 months after RYGB, respectively, whereas the median time to peak concentration was reduced by $50 \%$. Midazolam is a CYP3A4 substrate with a high extraction rate which was further increased after RYGB. Seaman et al. [57] developed an in vitro drug dissolution model to approximate the gastrointestinal environment of the preoperative and post RYGB states. Psychiatric medication tablets were placed in the two environments, and the weights of the dissolved portions were compared. Within the antidepressant class, the dissolution of citalopram and venlafaxine did not differ between conditions but antidepressant medications such as amitriptyline, fluoxetine, paroxetine, and sertraline dissolved less in the RYGB model than in the control model. With respect to anxiolytics and sedatives (buspirone, diazepam, lorazepam, trazodone, zolpidem), there was no difference in the two environments. The dissolution of the antipsychotics haloperidol and oxcarbazepine also did not vary between conditions. The newer antipsychotics (clozapine, olanzapine, quetiapine, risperidone, and ziprasidone) had lesser dissolution in the RYGB condition. Summarizing these results, it could be noted that the solubility of psychiatric tablets is altered after gastric bypass, but to demonstrate a change in the bioavailability of these drugs a clinical trial with monitoring of serum drug level is required.

The European Association for the Study of Obesity (EASO), the International Association for the Study of Obesity (IASO), the International Society for the Perioperative Care of the Obese Patient (ISPCOP), the Society American Gastrointestinal Endoscopic Surgeons (SAGES), 
the American College of Surgery (ACS), and the International Federation for the Surgery of Obesity and Metabolic Disorders (IFSO) have endorsed clinical practice guidelines for the perioperative nutritional, metabolic, and nonsurgical support of the bariatric surgery patient [58]. They recommended supplementation with multi-vitamins and oral calcium citrate for patients who had undergone RYGB and BPD. These practical guidelines do not give any recommendation as to how alter the choice or administration mode of these drugs after bariatric surgery.

\section{Oral Contraception and Bariatric Surgery}

In the normal population, oral contraceptives are dissolved in the stomach and transformed by bacterial enzymes and by enzymes in the intestinal mucosa. The metabolized and non-metabolized drugs are then absorbed through the intestinal mucosa and enter the portal vein blood [59]. The major estrogenic component of oral contraceptives, $17 \alpha$-ethinyloestradiol $\left(\mathrm{EE}_{2}\right)$, undergoes first-pass metabolism caused by gut wall sulfation at least $60 \%[60,61]$. Thus, absorption of oral contraceptives could be affected by malabsorptive and restrictivemalabsorptive bariatric procedures. Rapid weight loss in the months after bariatric surgery increases fertility while maternal and fetal risks from rapid weight loss remain elevated [62]. Consequently, effective contraception is critical in the postoperative period. Few data are published regarding the efficacy of oral contraception after bariatric surgery. A prospective study in 40 women (16-44 years) who underwent BPD evaluated the hormone status preoperatively and postoperatively after 2 and 7 days, 3 and 6 months, and 1 year [63]. The fertility and obstetric history was performed by sending a questionnaire at least 2 years after inclusion. From 9 patients using only an oral contraception, two patients developed an unintended pregnancy after BPD. Victor et al. [64] investigated the pharmacokinetics of two oral contraceptives (norethisterone (NET) $3 \mathrm{mg}$ and levonorgestrel (LNG) $0.25 \mathrm{mg}$ ) in 7 obese women (20-44 years) after jejunoileal bypass. The surgical procedure withdrew $37 \mathrm{~cm}$ of jejunum and $12.5 \mathrm{~cm}$ of ileum in continuity. Plasma concentrations of the two gestagens in the operated women were compared to those of a reference group before as well as $1,2,4,6,8$, and $24 \mathrm{~h}$ after ingestion of the tablet. The mean plasma levels of NET were lower in the operated patients at all times, except for the 24-hour samples, and those levels of LNG study, were significantly lower in the operated patients at 2,4 , and $6 \mathrm{~h}$. The authors concluded that the risk for contraceptive failure with low-dose gestagen mini-pills is increased in patients operated with jejunoileal bypass. More recently, Ciangura et al. [66] reported three cases of young women with etonorgestrel (ENG) implant after RYGB surgery. An implant (Implanon, Organon, France) containing $68 \mathrm{mg}$ of ENG had been inserted into inner side of the upper arm in anticipation of bariatric surgery. In two patients, the serum ENG concentrations remained higher than $150 \mathrm{pg} / \mathrm{ml} 6$ months after surgery with an effective contraceptive effect. In one patient, ENG concentrations were low at $125 \mathrm{pg} / \mathrm{ml}$ at 9 months, corresponding to the lower range of ENG concentrations observed after 3 years of use in normal-weight women [66]. The authors suggested that ENG-releasing implants might represent a safe contraceptive method in women undergoing bariatric surgery.

\section{Non-Oral Contraception}

There is consensus that pregnancy should be avoided for 12-24 months after bariatric surgery [17]. In women undergoing bariatric surgery, the use of contraceptive implants seemed to outperform their oral application. The American Congress of Obstetricians and 
Table 1. Partial recommendations for medical conditions added to the US medical eligibility criteria for contraceptive use [72]

\begin{tabular}{lllllll}
\hline Type of procedure & COC,, R & POP & DMPA & Implant & LNG IUD & Cu IUD \\
\hline Restrictive & 1 & 1 & 1 & 1 & 1 & 1 \\
Malabsorptive & COC: 2 ; P: 1 & 2 & 1 & 1 & 1 & 1
\end{tabular}

$\mathrm{COC}=$ Combined oral contraception; $\mathrm{P}=$ patch; $\mathrm{R}=$ ring; $\mathrm{POP}=$ progestin-only pills; DMPA: depot medroxyprogesterone acetate injection; LNG IUD = levonorgestrel intrauterine device; $\mathrm{Cu}$ IUD = copperbearing intrauterine device; 1 = a condition for which there is no restriction for the use of contraceptive method; 2 = a condition for which the theoretical or proven risks usually outweigh the advantages of using the method.

Gynecologists encouraged considering long-acting reversible contraception methods such as intrauterine devices (IUDs) and contraceptive implants for preventing unintended pregnancy in adolescents [67]. At pre-bariatric visits patients should be informed about these aspects, and patients should be encourage to make use of these devices. In terms of safety and efficacy, the LNG-releasing IUD appeared to have a favorable profile compared to alternatives [68]. The hormonal IUDs are highly efficacious, with less pregnancy rates than the copper IUDs [69]. However, discontinuation of treatment has been reported due to dysmenorrhea, pain, device expulsion, pelvic inflammatory disease, and infection [70, 71].

\section{Discussion and Conclusion}

It is important to keep in mind that there are only very little data in the scientific literature on the pharmacokinetics of drugs following bariatric surgery. Thus, each drug will have to be evaluated with respect to its site of absorption and its mechanism of absorption by means of certain enzymes or transporters, with special attention on parameters influencing the effectiveness of the absorption processes. The oral bioavailability of drugs after bariatric surgery should be examined for each drug separately. Considering the increase in the number of bariatric surgical interventions such as RYGB, the impact of these procedures on pharmacokinetics of drugs needs a thorough and detailed examination to guide therapeutic drug monitoring in obese patients after bariatric surgery. Especially, a potential impact of bariatric surgery on the efficacy of oral contraceptives is a matter of particular interest in order to effectively avoid unintended pregnancy over 12- to 24-month period post bariatric surgery. Bariatric surgery could potentially result in a transient change in drug absorption necessitating a change in the mode of contraception. Additional information on how bariatric surgery impacts drug pharmacokinetics and how this should affect clinical decisions is needed (table 1).

\section{Disclosure Statement}

The author has not declared any conflicts of interest. 
Schlatter et al.: Oral Contraceptives after Bariatric Surgery

\section{References}

1 World Health Organization: Obesity and Overweight, Fact sheet $\mathrm{N}^{\circ} 311$, January 2015. www.who.int/mediacentre/factsheets/fs311/en/ (last accessed March 27, 2017).

2 Weiss JL, Malone FD, Emig D, et al: Obesity, obstetric complications and cesarean delivery rate - a populationbased screening study. Am J Obstet Gynecol 2004;190:1091-1097.

-3 Bergholt T, Lim LK, Jørgensen JS, et al: Maternal body mass index in the first trimester and risk of cesarean delivery in nulliparous women in spontaneous labor. Am J Obstet Gynecol 2007;196:163.e1-5.

-4 Ogden CL, Carroll MD, Kit BK, et al: Prevalence of childhood and adult obesity in the United States, 2011-2012. JAMA 2014;311:806-814.

5 Ogden CL, Carroll MD, Curtin LR, et al: Prevalence of high body mass index in US children and adolescents, 2007-2008. JAMA 2010;303:242-249.

6 Cnattingius S, Bergström R, Lipworth L, et al: Prepregnancy weight and the risk of adverse pregnancy outcomes. N Engl J Med 1998;338:147-152.

$\checkmark 7$ Bellamy L, Casas JP, Hingorani AD, et al: Type 2 diabetes mellitus after gestational diabetes: a systematic review and meta-analysis. Lancet 2009;3731773-1779.

8 Singh J, Huang CC, Driggers RW, et al: The impact of pre-pregnancy body mass index on the risk of gestational diabetes. J Matern Fetal Neonatal Med 2012;25:5-10.

-9 Surkan PJ, Hsieh CC, Johansson AL, et al: Reasons for increasing trends in large for gestational age births. Obstet Gynecol 2004;104:720-726.

10 Buchwald H, Avidor Y, Braunwald E, et al: Bariatric surgery: a systematic review and meta-analysis. JAMA 2004;292:1724-1737.

11 Santry HP, Gillen DL, Lauderdale DS: Trends in bariatric surgical procedures. JAMA 2005;294:1909-1917.

12 Sjöström L, Lindroos AK, Peltonen M, et al: Lifestyle, diabetes, and cardiovascular risk factors 10 years after bariatric surgery. N Engl J Med 2004;351:2683-2693.

13 Belle SH, Berk PD, Courcoulas AP, et al: Safety and efficacy of bariatric surgery: Longitudinal Assessment of Bariatric Surgery. Surg Obes Relat Dis 2007;3:116-126.

14 Maggard MA, Yermilov I, Li Z, Maglione M, et al: Pregnancy and fertility following bariatric surgery: a systematic review. JAMA 2008;300:2286-2296.

15 Guelinckx I, Devlieger R, Vansant G: Reproductive outcome after bariatric surgery: a critical review. Hum Reprod Update 2009;15:189-201.

16 Haute Autorité de Santé (HAS): Clinical Practice Guidelines: Obesity Surgery in Adults. January 2009. www. has-sante.fr/portail/upload/docs/application/pdf/2010-11/obesity_surgery_-_guidelines.pdf (last accessed March 27, 2107).

17 American College of Obstetricians and Gynecologists (ACOG): ACOG Committee Opinion number 315, September 2005. Obesity in pregnancy. Obstet Gynecol 2005;106:671-675.

18 Eschwege E, Basdevant A, Crine A, et al: Type 2 diabetes mellitus in France in 2012:results from the ObEpi survey. Diabetes Metab 2015;41:55-61.

19 Miller AD, Smith KM: Medication and nutrient administration considerations after bariatric surgery. Am J Health Syst Pharm 2006;63:1852-1857.

20 Padwal R, Brocks D, Sharma A: A systematic review of drug absorption following bariatric surgery and its theoretical implications. Obes Rev 2010;11:41-50.

21 Buchwald H, Oien DM: Metabolic/bariatric surgery worldwide 2011. Obes Surg 2013;23:427-436.

-22 Kansou G, Lechaux D, Delarue J, et al: Laparoscopic sleeve gastrectomy versus laparoscopic mini gastric bypass: One year outcomes. Int J Surg 2016;22:18-22.

23 Schroeder R, Harrison TD, McGraw SL: Treatment of adult obesity with bariatric surgery. Am Fam Physician 2016;93:31-37.

24 Li P, Fu P, Chen J, et al: Laparoscopic Roux-en-Y gastric bypass vs. laparoscopic sleeve gastrectomy for morbid obesity and diabetes mellitus: a meta-analysis of sixteen recent studies. Hepatogastroenterology 2013;60: 132-137.

25 Moloney BM, Hynes DA, Kelly ME, et al: The role of laparoscopic sleeve gastrectomy as a treatment for morbid obesity; review of outcomes. Ir J Med Sci 2017;186:143-149.

26 Ruiz-Tovar J, Llavero C, Zubiaga L, et al: Maintenance of multivitamin supplements after sleeve gastrectomy. Obes Surg 2016;26:2324-2430.

27 Custodio JM, Wu CY, Benet LZ: Predicting drug disposition, absorption/elimination/transporter interplay and the role of food on drug absorption. Adv Drug Deliv Rev 2008;60:717-733.

28 Russell TL, Berardi RR, Barnett JL, et al: pH-related changes in the absorption of dipyridamole in the elderly. Pharm Res 1994;11:136-143.

29 Blum RA, D'Andrea DT, Florentino BM, et al: Increased gastric pH and the bioavailability of fluconazole and ketoconazole. Ann Intern Med 1991;114:755-757.

-30 Charman WN, Porter CJ, Mithani S, et al: Physiochemical and physiological mechanisms for the effects of food on drug absorption: the role of lipids and pH. J Pharm Sci 1997;86:269-282.

-31 Yamashita S, Kataoka M, Higashino H, Set al: Measurement of drug concentration in the stomach after intragastric administration of drug solution to healthy volunteers: analysis of intragastric fluid dynamics and drug absorption. Pharm Res 2013;30:951-958. 
32 Lahner E, Annibale B, Delle Fave G: Systematic review: impaired drug absorption related to the co-administration of antisecretory therapy. Aliment Pharmacol Ther 2009;29:1219-1229.

-33 Mitra A, Kesisoglou F: Impaired drug absorption due to high stomach pH: a review of strategies for mitigation of such effect to enable pharmaceutical product development. Mol Pharm 2013;10:3970-3979.

34 Ishida RK, Faintuch J, Paula AM, et al: Microbial flora of the stomach after gastric bypass for morbid obesity. Obes Surg 2007;17:752-758.

35 Hogben CM, Schenker LS, Tocco DJ, et al: Absorption of drugs from the stomach II: the human. J Pharmacol Exp Ther 1957;120:540-545.

-36 Glaeser H, Bailey DG, Dresser GK, et al: Intestinal drug transporter expression and the impact of grapefruit juice in humans. Clin Pharmacol Ther 2007;81:362-370.

-37 Maeda T, Takahashi K, Ohtsu N, et al: Identification of influx transporter for the quinolone antibacterial agent levofloxacin. Mol Pharm 2007;4:85-94.

-38 Inui K, Tomita Y, Katsura T, et al: $\mathrm{H}+$ coupled active transport of bestatin via the dipeptide transport system in rabbit intestinal brush-border membranes. J Pharmacol Exp Ther 1992;260:482-486.

-39 Walter E, Kissel T, Reers M, Dickneite G, et al: Transepithelial transport properties of peptidomimetic thrombin inhibitors in monolayers of a human intestinal cell line (Caco-2) and their correlation to in vivo data. Pharm Res 1995;12:360-365.

40 Adibi SA: Regulation of expression of the intestinal oligopeptide to transport (Pept-1) in health and disease. Am J Physiol Gastrointest Liver Physiol 2003;285:G779-788.

41 Adibi SA: The oligopeptide to transport (Pept-1) in human internal: biology and function. Gastroenterology 1997;113:332-340.

42 Matheny CJ, Lamb MW, Brouwer KR, et al: Pharmacokinetic and pharmacodynamic implications of P-glycoprotein modulation. Pharmacotherapy 2001;21:778-796.

43 Mouly S, Paine MF: P-glycoprotein increases from proximal to distal regions of human small intestine. Pharm Res 2003;20:1595-1599.

44 Paine MF, Khalighi M, Fisher JM, et al: Characterization of interintestinal and intraintestinal variations in human CYP3A-dependent metabolism. J Pharmacol Exp Ther 1997;283:1552-1562.

$\checkmark 45$ Darwich AS, Pade D, Ammori BJ, et al: With mechanistic pharmacokinetic model to assess modified oral drug bioavailability post bariatric surgery in morbidly obese patients: interplay between CYP3A gut wall metabolism, permeability and dissolution. J Pharm Pharmacol 2012;64:1008-1024.

$\$ 46$ Fleisher D, Li C, Zhou Y, Pao LH, et al: Drug, meal and formulation interactions influencing drug absorption after oral administration. Clinical implications. Clin Pharmacokinet 1999;36:233-254.

$\$ 47$ Tesserommatis C, Saranteas T, Chatzijanni E, et al: Modifications off clonidine binding to rabbit liver protein under the influence off not-steroid-anti-inflammatory drugs in vitro. Eur J Drug Metab Pharmacokinet 2003; 28:245-247.

-48 Terry SI, Gould JC, McManus JP, et al: Absorption of penicillin and paracetamol after small intestinal bypass surgery. Eur J Clin Pharmacol 1982;23:245-248.

-49 Pang SK: Modeling off intestinal drug absorption: roles off transporters and metabolic enzymes (for the Gillette Review Series). Drug Metab Dispos 2003;31:1507-1519.

50 Arakawa H, Shirasaka Y, Haga M, et al: Active intestinal absorption of fluoroquinolone antibacterial agent ciprofloxacin by organic anion transporting polypeptide, Oatp1a5. Biopharm Drug Dispos 2012;33:332-341.

51 Colin P, Eleveld DJ, Struys MM, et al: Moxifloxacin dosing in post-bariatric surgery patients. Br J Clin Pharmacol 2014;78:84-93.

-52 Magee SR, Shih G, Hume A: Malabsorption off oral antibiotics in pregnancy after gastric bypass surgery. J Am Board Fam Med 2007;20:310-313.

-53 Hamilton R, Thai XC, Ameri D, et al: Oral bioavailability of linezolid before and after Roux-en-Y gastric bypass surgery: is dose modification necessary in obese subjects? J Antimicrob Chemother 2013;68:666-673.

54 Chan LN, Lin YS, Tay-Sontheimer JC, et al: Proximal Roux-en-Y gastric bypass alters drug absorption pattern but not systemic exposure of CYP3A4 and P-glycoprotein substrates. Pharmacotherapy 2015;35:361-369.

55 Irwin AN, McCool KH, Delate T, et al: Assessment of warfarin dosing requirements after bariatric surgery in patients requiring long-term warfarin therapy. Pharmacotherapy 2013;33:1175-1183.

56 Smith A, Henriksen B, Cohen A: Pharmacokinetic considerations in Roux-en-Y gastric bypass patients. Am J Health-Syst Pharm 2011;68:2241-2247.

57 Seaman JS, Bowers SP, Dixon P, et al: Dissolution off common psychiatric medications in has Rouen there gastric bypass model. Psychosomatics 2005;46:250-253.

58 Mechanick JI, Youdim A, Jones DB, et al: Clinical practice guidelines for the perioperative nutritional, metabolic, and nonsurgical support of the bariatric surgery patient - 2013 update: cosponsored by American Association of Clinical Endocrinologists, the Obesity Society, and American Society for Metabolic \& Bariatric Surgery. Surg Obes Relat Dis 2013;9:159-191.

-59 Edelman AB, Cherala G, Stanczyk FZ: Metabolism and pharmacokinetics of contraceptive steroids in obese women: a review. Contraception 2010;82:314-323.

60 Belle DJ, Callaghan JT, Gorski JC, et al: The effects of an oral contraceptive containing ethinyloestradiol and norgestrel on CYP3A activity. Br J Clin Pharmacol 2002;53:67-74.

61 Back DJ, Breckenridge AM, MacIver M, et al: The gut wall metabolism of ethinyloestradiol and its contribution to the pre-systemic metabolism of ethinyloestradiol in humans. Br J Clin Pharmacol 1982;13:325-330. 
62 Saber AA, Elgamal MH, McLeod MK: Bariatric surgery: the past, present, and future. Obes Surg 2008;18:121128.

63 Gerrits EG, Ceulemans R, van Hee R: Contraceptive treatment after biliopancreatic diversion needs consensus. Obes Surg 2003;13:378-382.

64 Victor A, Odlind V, Kral JG: Oral contraceptive absorption and sex hormone binding globulins in obese women: effects of jejunoileal bypass. Gastroenterol Clin North Am 1987;16:483-491.

65 Ciangura C, Corigliano N, Basdevant A, et al: Etonorgestrel concentrations in morbidly obese women following Roux-en-Y gastric bypass surgery: three case reports. Contraception 2011;84:649-651.

66 Huber J: Pharmacokinetics of Implanon. An integrated analysis. Contraception 1998;58(6 suppl):85S-90S.

-67 American College of Obstetricians and Gynecologists (ACOG): Committee Opinion No. 392, December 2007. Intrauterine device and adolescents. Obstet Gynecol 2007;110:1493-1495.

68 Hillman JB, Miller RJ, Inge TH: Adolescent menstrual concerns and intrauterine contraception among bariatric surgery patient. J Womens Health 2011;20:533-538.

69 Heinemann K, Reed S, Moehner S, Minh TD: Comparative contraceptive effectiveness of levonorgestrelreleasing and copper intrauterine devices: the European Active Surveillance Study for Intrauterine Devices. Contraception 2015;91:280-283.

70 Rowe P, Farley T, Peregoudov A, et al: Safety and efficacy in parous women of a 52-mg levonorgestrel-medicated intrauterine device: a 7-year randomized comparative study with the TCu380A. Contraception 2016;93: 498-506.

71 Steenland MW, Zapata LB, Brahmi D, et al: Appropriate follow up to detect potential adverse events after initiation of select contraceptive methods: a systematic review. Contraception 2013;87:611-624.

72 Jacobson JC, Aikins Murphy P: United States medical eligibility criteria for contraceptive use 2010: a review of changes. J Midwifery Womens Health 2011;56:598-607. 